\title{
Serum profiles of tryptophan-kynurenine pathway metabolites in psoriasis
}

\author{
Mariko Seishima $^{1,2}{ }^{*}$, Yasuko Yamamoto ${ }^{3}$, Masashi Sakurai ${ }^{3}$, Rika Sakai $^{3}$, Kento Fujii ${ }^{1}{ }^{\oplus}$, Yoko Mizutani $^{1}$, \\ Kuniaki Saito ${ }^{3}$ \\ ${ }^{1}$ Department of Dermatology, Gifu University Graduate School of Medicine, Yanagido 1-1, Gifu 501-1194, Japan \\ ${ }^{2}$ Department of Dermatology, Asahi University Hospital, Hashimoto-cho 3-23, Gifu 500-8523, Japan \\ ${ }^{3}$ Department of Disease Control and Prevention, Fujita Health University Graduate School of Health Sciences, 1-98 \\ Dengakugakubo, Kutsukake, Toyoake, Aichi 470-1192, Japan
}

*Correspondence: Mariko Seishima, Department of Dermatology, Gifu University Graduate School of Medicine, Yanagido 1-1, Gifu 501-1194, Japan. marikoseishima@yahoo.co.jp

Academic Editor: Masutaka Furue, Kyushu University, Japan

Received: July 17, 2021 Accepted: August 30, 2021 Published: October 31, 2021

Cite this article: Seishima M, Yamamoto Y, Sakurai M, Sakai R, Fujii K, Mizutani Y, et al. Serum profiles of tryptophan-kynurenine pathway metabolites in psoriasis. Explor Immunol. 2021;1:258-68. https://doi.org/10.37349/ei.2021.00017

\section{Abstract}

Aim: Chronic inflammation is closely associated with tryptophan (TRP)-kynurenine (KYN) metabolic pathway. However, TRP-KYN pathway has not been fully elucidated in psoriasis, a systemic inflammatory disease with skin lesions and extracutaneous manifestations. Herein, we studied comprehensively serum profiles of TRP-KYN pathway metabolites in psoriatic patients (PSOs) to clarify the involvement of this pathway in the pathophysiology of psoriasis and to evaluate serum biomarkers reflecting systemic inflammation in PSOs.

Methods: The concentrations of main TRP metabolites, TRP, KYN, 3-hydroxykynurenine (3HK), kynurenic acid (KYNA), 3-hydroxyanthranilic acid (3HAA), and anthranilic acid (AA), were determined by high-performance liquid chromatography in the sera from 65 PSOs and 35 healthy controls (HCs). The levels of these metabolites and the ratios of metabolites were compared between these subjects. The correlations between these values and the psoriasis area severity index (PASI) scores were analyzed. Skin samples from PSOs and HCs were subjected to immunohistochemical staining for kynureninase, catabolic enzyme from KYN or $3 \mathrm{HK}$ to downstream. Cytokine concentrations were comprehensively measured in the same samples and the correlations between the cytokine levels and TRP-KYN pathway metabolite levels were examined.

Results: Serum TRP, KYN, and KYNA concentrations were lower and the 3HAA concentrations were higher in PSOs than in HCs. The ratios of 3HK/KYN, 3HAA/3HK, and 3HK/AA were higher in PSOs than in HCs. The AA levels and the ratio of AA/KYN were weakly positively correlated, and TRP, KYNA, and 3HK levels and the ratios of KYNA/KYN and 3HAA/AA were weakly negatively correlated with the PASI scores. The $\mathrm{AA}, \mathrm{KYN}$, and KYNA levels were positively correlated with the interferon gamma-induced protein 10 (IP-10) concentrations. Kynureninase expression was enhanced in the epidermis, both involved and uninvolved skin. Conclusions: Serum profiles of TRP-KYN pathway metabolites differed between PSOs and HCs. TRP-KYN pathway-associated processes, including kynureninase activation, may be involved in the pathogenesis of psoriasis, and thus serve as targets for psoriasis therapy. 


\section{Keywords}

Psoriasis, kynureninase, kynurenine metabolite, tryptophan-kynurenine pathway, indoleamine 2,3-dioxygenase

\section{Introduction}

Psoriasis is a chronic inflammatory skin disease characterized by the thickening of the epidermis and skin infiltration of polymorphonuclear cells. The tumor necrosis factor (TNF)- $\alpha /$ interleukin (IL)-23/IL-17A axis plays a key role in the induction and progression of psoriasis; thus, biological drugs againstTNF- $\alpha /$ IL-23/IL-17A show a good therapeutic efficacy $[1,2]$.

Various comorbidities such as cardiovascular disease, metabolic syndrome, inflammatory bowel disease, and depression are associated with psoriasis [3]. It has been proposed that shared inflammatory pathways may act as a driving force for both psoriasis and extracutaneous manifestations. Thus, a paradigm shift in concept of the pathophysiology of psoriasis has occurred; psoriasis is now viewed as a systemic inflammatory condition and not merely a "skin-deep" condition [4].

Tryptophan (TRP) metabolism pathway is strongly associated with immune suppression [5-7]. TRP is oxidized by cleavage of the indole ring moiety, and kynurenine (KYN) is generated. This pathway is initiated either by tryptophan 2,3-dioxagenase (TDO), indoleamine 2,3-dioxygenase (ID0)1, or IDO2 (Figure 1). TDO is almost exclusively expressed in the liver and is induced in some cell types such as leukocytes and endothelial cells by TRP levels or corticosteroids [8]. ID01 is the predominant enzyme extra-hepatically and is expressed in a lot of cell types including macrophages and keratinocytes $[5,6,9,10]$. ID01 is induced by various inflammatory cytokines such as interferon (IFN)- $\gamma$, and is upregulated in several inflammatory conditions including psoriasis [5, 11]. ID02, an isotype enzyme of ID01, shows similar structural and enzymatic activities as ID01, but differs in its expression pattern, substrate specificity, and downstream signaling pathways [12].

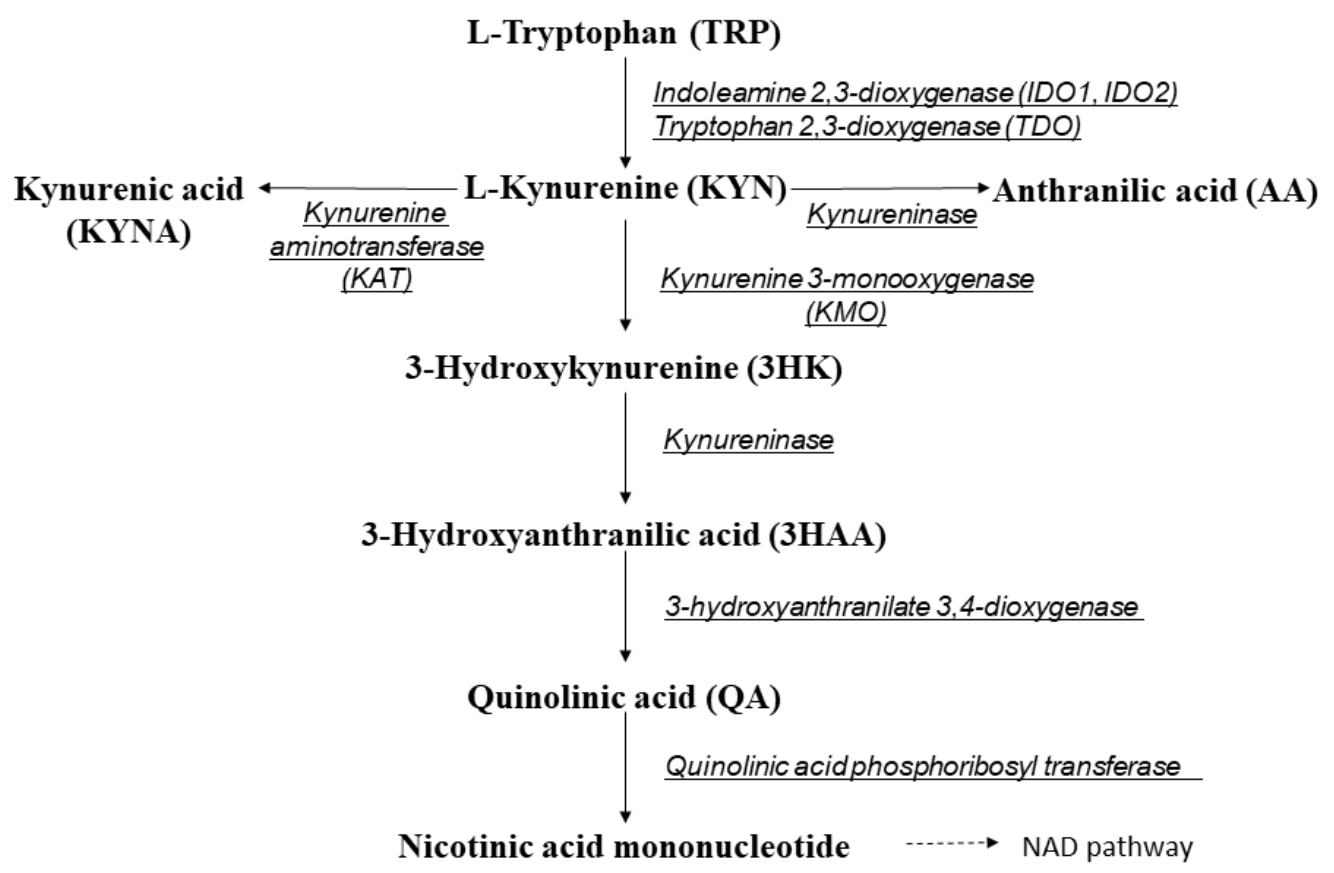

Figure 1. The KYN pathway of TRP degradation. NAD: nicotinamide adenine dinucleotide

Kynureninase is an enzyme in the TRP-KYN metabolism pathway that metabolizes the KYN and 3-hydroxykynurenine (3HK) into anthranilic acid (AA) and 3-hydroxyanthranilic acid (3HAA), respectively. Among several key genes elucidated by microarray and genomic studies [13], kynureninase is one gene prominently upregulated in psoriasis [14-18]. It is highly upregulated in involved psoriatic skin compared to uninvolved skin, though which is still upregulated compared to the normal skin [5]. 
The TRP-KYN metabolism pathway [19] produces various metabolites, such as KYN, 3HK, kynurenic acid (KYNA), 3HAA, AA, and quinolinic acid (QA) (Figure 1). In the present study, the serum profiles of TRP-KYN pathway metabolites were comprehensively examined in psoriatic patients (PSOs) to verify the involvement of this pathway in the pathophysiology of psoriasis and to evaluate the serum profiles of new psoriasis-related biomarkers that reflect systemic inflammation in PSOs. In addition, we aimed to assess the ratios of TRP-KYN pathway metabolites as indices of two main enzymes, ID01 and kynureninase, in this pathway.

\section{Materials and methods}

\section{Patients}

This study was approved by the ethical committee of Gifu University and was performed in strict adherence with the Declaration of Helsinki principles (2018-1949). After obtaining written informed consent, sera and skin biopsy specimens were collected from healthy controls (HCs) and PSOs. The patients were diagnosed as psoriasis clinically and histologically in Gifu University Hospital. The sera were stored at $-80^{\circ} \mathrm{C}$ before use.

A total of 65 PSOs (19 women and 46 men) and 35 HCs (11 women and 24 men) were included. The mean age of the PSOs was $58.2 \pm 13.7$ (women: $56.3 \pm 15.8$, men: $59.0 \pm 12.8$ ). The mean age of HCs was 56.9 \pm 12.5 (women: $57.5 \pm 12.1$, men: $56.6 \pm 12.9$ ). The patients showed different types of psoriasis: 51 patients with psoriasis vulgaris, 11 patients with psoriatic arthritis (PsA), and 3 patients with pustular psoriasis. The average psoriasis area severity index (PASI) score, as evaluated by the dermatologists was $4.2 \pm 6.4$ (women: $4.0 \pm 4.8$ and men: $4.3 \pm 6.9$ ) at the time of blood sample collection. The PASI scores were $4.33 \pm 4.8$ for patients with psoriasis vulgaris, $3.35 \pm 4.2$ for patients with PsA, and $8.3 \pm 3.0$ for patients with pustular psoriasis.

All PSOs were treated; 43 patients with biologics ( 5 with TNF- $\alpha$ inhibitors, 19 with IL-17 inhibitors, and 19 with IL-23 inhibitors), 10 patients with apremilast, 3 patients with retinoid, 1 patient with cyclosporine, and 8 patients with only topical treatments (8 patients with topical steroid + vitamin $\mathrm{D}_{3}$ and 2 patients with topical steroid only).

\section{Measurement of TRP-KYN pathway metabolites in sera}

The levels of TRP metabolites were determined according to our previous report [20]. Briefly, the levels of TRP, KYN, KYNA, AA, 3HK, and 3HAA were measured by high-performance liquid chromatography (HPLC) (SHIMAZU, Kyoto, Japan).

\section{Immunohistochemistry}

Skin specimens were obtained from the involved and uninvolved skin of four PSOs and from the skin of four HCs. Paraffin-embedded specimens were prepared using standard methods and the tissue sections were stained with hematoxylin and eosin and immunohistochemically using the antibodies against kynureninase (GeneTex, CA, USA) and Dako Envision System-labeled Polymer, HRP (Dako, Tokyo, Japan).

\section{Measurement of cytokines and chemokines in sera}

The serum levels of the following cytokines were determined using a MILLIPLEX multiplex assay system (Millipore, Billerica, MA) according to our previous report [20]: soluble CD40 ligand (sCD40L), epidermal growth factor (EGF), eotaxin, fibroblast growth factor (FGF)-2, Fms-related tyrosine kinase 3 ligand (FLT3-L), fractalkine, granulocyte colony-stimulating factor (G-CSF), granulocyte-macrophage colony-stimulating factor (GM-CSF), growth related oncogene- $\alpha$ (GRO- $\alpha$ ), chemokine (C-X-C motif) ligand (CXCL)1, IFN- $\alpha 2$, IFN- $\gamma$, IL-1 $\alpha$, IL-1 $\beta$, interleukin-1 receptor antagonist (IL-1RA), IL-2, IL-4, IL-5, IL-6, IL-7, IL-8, IL-9, and IL-10. Furthermore, the serum levels of IL-12 (p40), IL-12 (p70), IL-13, IL-15, IL-17A, IL-17E, IL-17F, IL-18, IL-22, IL-27, interferon gamma-induced protein 10 (IP-10), monocyte chemoattractant protein (MCP)-1, MCP-3, macrophage-derived chemokine [MDC, CC chemokine ligand (CCL)22], monokine induced by gamma interferon (MIG), CXCL9, macrophage inflammatory protein (MIP)-1 $\alpha$, MIP-1 $\beta$, platelet-derived growth factor (PDGF)-AA, PDGF-AB/BB, transforming growth factor (TGF)- $\alpha$, TNF- $\alpha$, TNF- $\beta$, and vascular endothelial growth factor (VEGF)-A were measured using the same system. 


\section{Statistical analysis}

The obtained values were expressed as the means \pm standard deviations (SDs). Pearson's product moment correlation coefficients and their statistical significance were calculated to determine the correlations between the quantified parameters. Welch's $t$ test was used to compare the data of TRP-KYN pathway metabolites from the unpaired groups. Wilcoxon test was used to compare the data of cytokines from the unpaired groups. Statistical significance was defined as a $P$-value of $<0.05$.

\section{Results}

\section{Measurement of TRP-KYN pathway metabolites in sera}

Serum TRP, KYN, and KYNA concentrations were significantly lower in PSOs than in the HCs. On the other hand, the 3HAA concentrations were higher in the PSOs than in the HCs, and the AA and 3HK levels showed no differences between the two groups (Table 1). The serum TRP-KYN pathway metabolite profiles did not show sex-associated differences, and no difference was observed in the profiles between the patients treated with various biologics and non-biologics (apremilast). The ratios of the 3HK to KYN, 3HAA to 3HK, and 3HAA to AA were significantly increased in PSOs, but the KYN/TRP, KYNA/KYN, and AA/KYN ratios between the PSOs and HCs were not different (Table 2).

Table 1. Differences in the serum concentrations of TRP-KYN pathway metabolites between HCs and PSOs

\begin{tabular}{llll}
\hline Metabolites & HCs (mean \pm SD) & PSOs (mean \pm SD) & $* *$ \\
\hline TRP $(\mu \mathrm{M})$ & $52.9 \pm 7.26$ & $48.0 \pm 8.34$ & $*$ \\
KYN $(\mu \mathrm{M})$ & $2.00 \pm 0.49$ & $1.73 \pm 0.47$ & $*$ \\
KYNA $(\mathrm{nM})$ & $37.6 \pm 9.81$ & $32.0 \pm 16.8$ & \\
AA $(\mathrm{nM})$ & $17.1 \pm 6.78$ & $16.0 \pm 12.2$ & $* 18.6 \pm 8.76$ \\
3HK $(\mathrm{nM})$ & $18.8 \pm 12.9$ & $14.2 \pm 6.22$ & $*$ \\
3HAA $(\mathrm{nM})$ & $7.56 \pm 4.52$ & & \\
\hline
\end{tabular}

${ }^{*} P<0.05 ;{ }^{* *} P<0.01 ;{ }^{* * *} P<0.001$ vs. HCs

Table 2. Differences in the ratio of TRP-KYN pathway metabolites between HCs and PSOs

\begin{tabular}{|c|c|c|c|}
\hline Ratios of metabolites & HCs (mean \pm SD) & PSOs (mean \pm SD) & \\
\hline KYN/TRP & $0.0382 \pm 0.0102$ & $0.0369 \pm 0.0121$ & \\
\hline KYNA/KYN & $0.0196 \pm 0.0060$ & $0.0188 \pm 0.0085$ & \\
\hline $\mathrm{AA} / \mathrm{KYN}$ & $0.0087 \pm 0.0027$ & $0.0092 \pm 0.0048$ & \\
\hline $3 \mathrm{HK} / \mathrm{KYN}$ & $0.0093 \pm 0.0039$ & $0.0111 \pm 0.0052$ & * \\
\hline 3HAA/3HK & $0.4319 \pm 0.2317$ & $0.9026 \pm 0.4619$ & $* * *$ \\
\hline 3HAA/AA & $0.499 \pm 0.385$ & $1.0893 \pm 0.614$ & *** \\
\hline
\end{tabular}

${ }^{*} P<0.05 ;{ }^{* *} P<0.001$ vs. HCs

The AA levels showed a weakly positive correlation, but the TRP, KYNA, and 3HK levels were weakly negatively correlated with the PASI scores; the KYN and 3HAA levels showed no correlation with the PASI scores. The AA/KYN ratio showed a weakly positive correlation, and the KYNA/KYN and 3HAA/AA ratios were weakly negatively correlated with the PASI score (Table 3).

\section{Immunohistochemistry}

Kynureninase was stained in the epidermis of psoriatic involved skin more strongly than in that of the skin from the HCs (Figure 2). It was also distributed in the epidermis of uninvolved skin of psoriasis patients. 
Table 3. Pearson's correlation coefficients between PASI score and the TRP-KYN pathway metabolites in PSOs

\begin{tabular}{ll}
\hline Metabolites and ratios & Correlation coefficient \\
\hline TRP & -0.28 \\
KYN & -0.01 \\
KYNA & -0.29 \\
AA & 0.38 \\
3HK & -0.27 \\
3HAA & -0.07 \\
KYN/TRP & 0.15 \\
KYNA/KYN & -0.33 \\
AA/KYN & 0.45 \\
3HK/KYN & -0.22 \\
3HAA/3HK & 0.21 \\
3HAA/AA & -0.26 \\
\hline
\end{tabular}

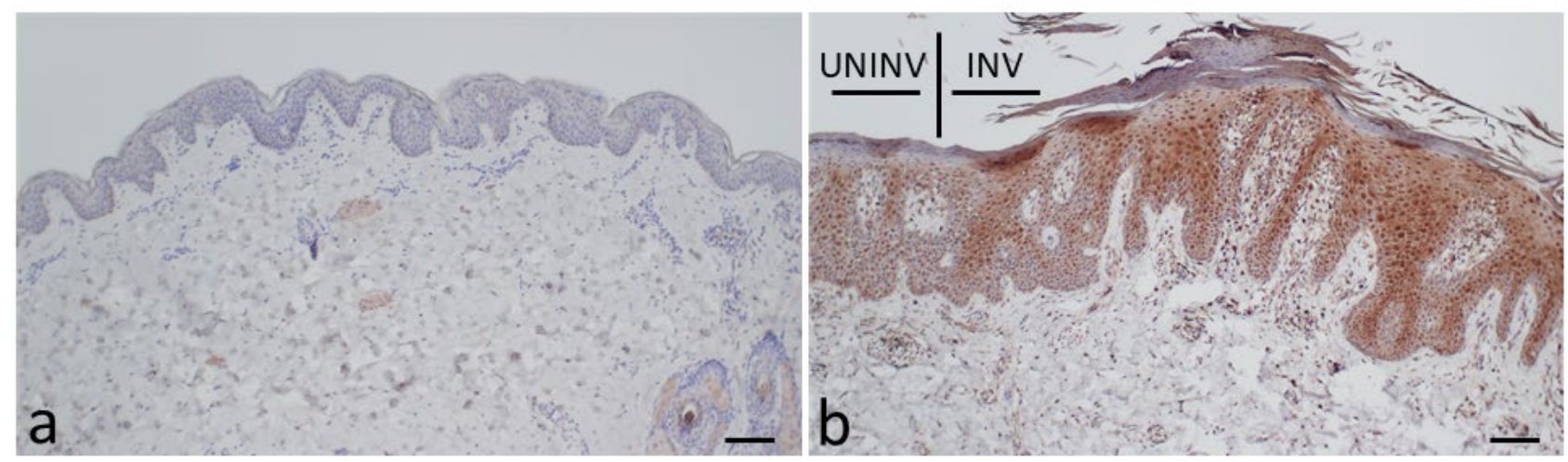

Figure 2. Histological distribution of the expression of kynureninase in psoriasis. (a) The skin from a HC; (b) the skin from a PSO. INV: involved skin; UNINV: uninvolved skin. The tissue sections were stained using the antibodies against kynureninase. Scale bars: $100 \mu \mathrm{m}$

\section{Cytokine and chemokine concentrations in sera}

The serum concentrations of FGF-2, FLT3-L, IFN- $\alpha 2$, IL-1 $\alpha$, IL-2, IL-5, IL-9, IL-17A, MCP-1, MDC, and TGF- $\alpha$ were increased in the sera from PSOs, compared to the sera from the HCs. On the other hand, the IL-17F and IP-10 concentrations in the sera from PSOs were lower than those in the sera from the HCs (Table 4). The AA, KYN, and KYNA levels were positively correlated with the IP-10 levels. The IL-1RA levels were weakly positively correlated, while the IL-9 levels were weakly negatively correlated with the PASI scores (Table 5).

\section{Discussion}

In the present study, the serum profiles of TRP-KYN pathway metabolites and the ratios of the metabolites were comprehensively evaluated in the PSOs. Consequently, the serum TRP, KYN, and KYNA concentrations were significantly lower, but the 3HAA concentrations were markedly higher in PSOs than in the HCs (Table 1). The ratios of $3 \mathrm{HAA}$ to $3 \mathrm{HK}, 3 \mathrm{HK}$ to KYN, and 3HAA to AA were significantly increased (Table 2). The TRP, KYNA, and 3HK levels were weakly negatively correlated with the PASI scores (Table 3). These results indicate that the TRP-KYN-3HK-3HAA metabolic pathway is upregulated in psoriasis. The concentrations of KYNA, which is present downstream of KYN, may be reduced following the decreased KYN concentrations. Although AA is also present downstream of KYN, the pathway from KYN to AA by kynureninase may be enhanced in the PSOs, resulting in no differences of AA levels between the PSOs and HCs. 
Table 4. Differences in the serum concentrations of cytokines and chemokines between HCs and PSOs

\begin{tabular}{|c|c|c|c|c|c|}
\hline & & & HCs (mean \pm SD; ng/L) & PSOs (mean \pm SD; ng/L) & \\
\hline \multirow[t]{43}{*}{ Pro-inflammatory } & sCD40L & & $25.28 \pm 16.06$ & $27.72 \pm 21.33$ & \\
\hline & EGF & & $38.45 \pm 36.81$ & $59.11 \pm 73.34$ & \\
\hline & Eotaxin & & $96.79 \pm 33.93$ & $105.2 \pm 46.51$ & \\
\hline & FGF-2 & $\Delta$ & $34.57 \pm 18.30$ & $38.95 \pm 76.69$ & * \\
\hline & FLT-3L & $\Delta$ & $1.35 \pm 1.55$ & $4.95 \pm 4.75$ & *** \\
\hline & Fractalkine & & $59.78 \pm 30.74$ & $57.96 \pm 41.50$ & \\
\hline & G-CSF & & $9.46 \pm 13.58$ & $19.09 \pm 70.55$ & \\
\hline & GM-CSF & & $2.26 \pm 1.63$ & $2.57 \pm 2.27$ & \\
\hline & GRO- $\alpha$ & & $20.93 \pm 20.60$ & $17.03 \pm 12.66$ & \\
\hline & IFN- $\alpha 2$ & $\boldsymbol{\Delta}$ & $8.08 \pm 2.18$ & $8.86 \pm 12.07$ & ** \\
\hline & IFN-Y & & $1.60 \pm 2.21$ & $3.75 \pm 7.57$ & \\
\hline & $\mathrm{IL}-1 \alpha$ & $\Delta$ & $4.86 \pm 2.08$ & $8.32 \pm 21.86$ & ** \\
\hline & $\mathrm{IL}-1 \beta$ & & $3.31 \pm 7.82$ & $3.99 \pm 8.81$ & \\
\hline & IL-2 & $\boldsymbol{\Delta}$ & $0.65 \pm 0.27$ & $0.79 \pm 1.56$ & ** \\
\hline & $\mathrm{IL}-4$ & & $1.57 \pm 1.36$ & $1.44 \pm 1.37$ & \\
\hline & IL-5 & $\Delta$ & $0.65 \pm 0.70$ & $1.49 \pm 2.67$ & * \\
\hline & IL-6 & & $1.44 \pm 2.89$ & $5.52 \pm 18.83$ & \\
\hline & IL-7 & & $10.12 \pm 9.19$ & $12.82 \pm 30.87$ & \\
\hline & IL-8 & & $7.88 \pm 2.35$ & $9.46 \pm 10.80$ & \\
\hline & IL-9 & $\Delta$ & $4.92 \pm 6.92$ & $10.21 \pm 9.85$ & $* *$ \\
\hline & IL-12p40 & & $27.75 \pm 19.19$ & $46.22 \pm 81.55$ & \\
\hline & IL-12p70 & & $3.39 \pm 1.33$ & $4.25 \pm 5.20$ & \\
\hline & IL-13 & & $8.86 \pm 8.28$ & $8.08 \pm 8.49$ & \\
\hline & IL-15 & & $2.93 \pm 0.93$ & $4.40 \pm 4.64$ & \\
\hline & IL-17A & $\Delta$ & $1.45 \pm 0.91$ & $3.05 \pm 10.24$ & * \\
\hline & IL-17E/IL-25 & & $143.8 \pm 120.4$ & $164.6 \pm 152.8$ & \\
\hline & IL-17F & $\nabla$ & $31.18 \pm 3.79$ & $22.04 \pm 22.40$ & *** \\
\hline & IL-18 & & $22.70 \pm 13.75$ & $21.67 \pm 18.09$ & \\
\hline & IL-22 & & $39.42 \pm 51.27$ & $39.82 \pm 44.67$ & \\
\hline & IL-27 & & $1082 \pm 519.6$ & $1282 \pm 914.5$ & \\
\hline & IP-10 & $\nabla$ & $219.1 \pm 122.3$ & $123.4 \pm 76.86$ & $* * *$ \\
\hline & MCP-1 & $\Delta$ & $335.3 \pm 70.54$ & $392.5 \pm 126.7$ & * \\
\hline & MCP-3 & & $10.88 \pm 6.72$ & $10.73 \pm 6.82$ & \\
\hline & MDC & $\Delta$ & $490.4 \pm 154.2$ & $663.0 \pm 367.4$ & $* *$ \\
\hline & MIG & & $1764 \pm 1250$ & $1812 \pm 1484$ & \\
\hline & MIP-1a & & $6.06 \pm 4.95$ & $10.61 \pm 13.38$ & \\
\hline & MIP-1 $\beta$ & & $32.66 \pm 14.50$ & $34.28 \pm 18.96$ & \\
\hline & PDGF-AA & & $2807 \pm 915.6$ & $2847 \pm 947.0$ & \\
\hline & PDGF-AB/BB & & $29323 \pm 50067$ & $11958 \pm 18352$ & \\
\hline & TGF- $\alpha$ & $\Delta$ & $2.60 \pm 2.86$ & $7.41 \pm 11.23$ & $* * *$ \\
\hline & TNF- $\alpha$ & & $25.08 \pm 15.16$ & $26.51 \pm 16.05$ & \\
\hline & TNF- $\beta$ & & $3.99 \pm 3.91$ & $3.53 \pm 3.30$ & \\
\hline & VEGF- $\alpha$ & & $267.8 \pm 181.9$ & $271.0 \pm 170.8$ & \\
\hline \multirow[t]{2}{*}{ Anti-inflammatory } & IL-1RA & & $3.23 \pm 2.06$ & $6.09 \pm 12.64$ & \\
\hline & IL-10 & & $2.42 \pm 2.52$ & $10.22 \pm 52.99$ & \\
\hline
\end{tabular}

${ }^{\star} P<0.05 ;{ }^{* \star} P<0.01 ;{ }^{* \star \star} P<0.001$ vs. HCs; $\boldsymbol{\Delta} / \nabla$ : significantly higher/lower in PSOs than in the HCs 
Table 5. Pearson's correlation coefficients $(|r|>0.3)$ between the TRP-KYN pathway metabolites and cytokine levels $(A)$ and between PASI score and cytokine levels (B) in PSOs

\begin{tabular}{llll}
\hline (A) & TRP-KYN pathway metabolites & Cytokines & Correlation coefficient \\
\hline AA & IP-10 & 0.64 \\
& KYN & IP-10 & 0.49 \\
& AA & FLT3-L & 0.38 \\
& TRP & EGF & 0.37 \\
& KYN & MIG & 0.37 \\
& AA & MDC & 0.32 \\
& AA & MIG & 0.32 \\
& TRP & MIG & -0.32 \\
& AA & MIP-1 & 0.31 \\
& 3HK & IL-17F & -0.31 \\
& KYNA & IP-10 & 0.30 \\
& AA & Fractalkine & 0.30 \\
& KYN & FLT3-L & 0.30 \\
\hline (B) & PASI score & Cytokines & Correlation coefficient \\
\cline { 2 - 3 } & PASI score & IL-1RA & 0.45 \\
& PASI score & IL-9 & -0.30 \\
\hline
\end{tabular}

ID01 and kynureninase have been reported as two main enzymes in TRP-KYN pathway. ID01 and kynureninase are upregulated in the involved skin, compared to the uninvolved skin of PSOs [5, 11, 21, 22]. ID01 acts as an immune modulator in immune cells of healthy conditions [23]. KYNA [24] and KYN generated by ID01 exert active immune-suppressive effects [7], through mechanisms such as inducing regulatory T cells via binding to the aryl-hydrocarbon receptor (AhR) $[25,26]$. There have not been fully studied about ID02 and TDO in psoriasis. IDO2 decreases IL-17 expression, resulting in the suppression of skin inflammation in imiquimod-induced psoriasis-like dermatitis [27]. In the present study, the TRP levels decreased in the PSOs, suggesting IDO1 activation. However, the KYN levels also decreased and the KYN/TRP ratio did not show the differences between the PSOs and HCs. These data may suggest activation of kynureninase and/or kynurenine 3-monooxygenase (KMO). As successful treatments to psoriasis reduce IDO1 and kynureninase [5], KYN/TRP ratio might be affected by various treatments in the present study. Otherwise, the activities of TDO or ID02 might be involved in the KYN generation in psoriasis.

The AA/KYN and 3HAA/3HK ratios were assessed as possible indices of kynureninase. The AA/KYN ratio and AA concentrations did not show a difference between the PSOs and HCs, but were positively correlated with PASI scores (Table 3), suggesting that the pathway from KYN to AA may be enhanced strongly in the PSOs with higher PASI scores, but not in those with lower PASI scores. The 3HAA/3HK ratio was significantly elevated in the PSOs than HCs, but was not correlated with PASI scores. It suggests that the pathway from $3 \mathrm{HK}$ to 3HAA, which is main reaction by kynureninase, is fully activated in the PSOs independently of PASI scores. As human kynureniase prefers $3 \mathrm{HK}$ to KYN as a substrate, the reaction rate may not be the same between KYN to AA and 3HK to 3HAA.

From these results, it is suggested that kynureninase activity is upregulated in PSOs. Therefore, the enhanced expression of kynureninase was confirmed histologically in the epidermis from psoriatic skin, both the involved and uninvolved skin, compared to the healthy skin (Figure 2). However, unfortunately, the kynureninase activity could not be determined in the psoriatic skin in this study.

The 3HAA/AA ratio has been reported to show possibly an inflammatory state, with a decrease indicating an anti-inflammatory response $[28,29]$. In the present study, the 3HAA/AA ratio was significantly higher in the PSOs than in HCs, and was weakly negatively correlated with PASI scores. Not only kynureninase but also the other enzymes such as KMO are involved in this metabolic pathway, and thus the activity of KMO might be altered in the PSOs. 
Several studies have determined the serum levels of cytokines and chemokines in PSOs, but the results are controversial [30,31]. It can be said that the correlation between the levels of TRP-KYN pathway metabolites and cytokines has not been fully understood. KYNA plays an important role in modulating the expression of IL23 and IL-17 in dendritic cells and Thelper 17 (Th17) cells [32]. The interaction between AhR and endogenous ligands such as KYN and KYNA is known to alter the inflammatory profiles of psoriasis [33]. Altered regulation of TRP-KYN metabolism may reduce the levels of AhR ligands [24, 34], resulting in the increased expression of proinflammatory cytokines in psoriatic lesions [35]. Although increased IP-10 expression has been reported in the epidermis of psoriasis [36,37], the serum IP-10 levels were significantly lower in PSOs than in HCs (Table 4) and were positively correlated with the AA, KYN, and KYNA levels (Table 5). It is suggested that some cytokines and chemokines including IP-10 play a role in the pathogenesis of psoriasis in association with TRP-KYN metabolism pathway. However, as the treatment for psoriasis varies in the present study, the correlation between cytokine levels and TRP-KYN pathway metabolites may be affected by the treatments.

TRP-KYN pathway metabolites present downstream of kynureninase upregulate several cytokines, chemokines, and cell adhesions-related factors. Of interest is that the dichotomous actions of TRP metabolism have been described in neurobiology, with early TRP metabolites such as KYN being considered to possess a neuroprotective role and late TRP metabolites, such as QA, demonstrating neurotoxic activity [38, 39]. Future studies that examine all the enzymes within this pathway will provide a clue to understand how this pathway is involved in the inflammation. In addition, the relationship between TRP-KYN metabolism and the immune system may be more complicated and remains to be ascertained in further studies. In addition, TRP metabolism is reported to modulate immune responses dichotomously with kynureninase as a switch between immunosuppressive and inflammatory outcomes, in not only psoriasis, but in other inflammatory diseases [5].

In conclusion, the serum profiles of TRP-KYN pathway metabolites differed between PSOs and HCs, suggesting that kynureninase activity is upregulated in PSOs compared to that in HCs. Limitations of our study are the small sample size and variety of treatments; hence, further studies are required to completely assess the involvement of TRP-KYN pathway metabolites in the pathogenesis of psoriasis and the outcome may give us the potential of these metabolites to serve as biomarkers for psoriasis.

\section{Abbreviations}

3HAA: 3-hydroxyanthranilic acid

3HK: 3-hydroxykynurenine

AA: anthranilic acid

AhR: aryl-hydrocarbon receptor

EGF: epidermal growth factor

FGF: fibroblast growth factor

FLT3-L: Fms-related tyrosine kinase 3 ligand

HCs: healthy controls

IDO: indoleamine 2,3-dioxygenase

IFN: interferon

IL: interleukin

IL-1RA: interleukin-1 receptor antagonist

IP-10: interferon gamma-induced protein 10

KMO: kynurenine 3-monooxygenase

KYN: kynurenine

KYNA: kynurenic acid

MCP: monocyte chemoattractant protein 
MDC: macrophage-derived chemokine

MIG: monokine induced by gamma interferon

MIP: macrophage inflammatory protein

PASI: psoriasis area severity index

PDGF: platelet-derived growth factor

PSOs: psoriatic patients

QA: quinolinic acid

TDO: tryptophan 2,3-dioxagenase

TGF: transforming growth factor

TNF: tumor necrosis factor

TRP: tryptophan

\section{Declarations}

Acknowledgments

The authors would like to thank Mrs. Kayoko Tanaka for assisting data collection.

\section{Author contributions}

Seishima M and KS contributed conception and design of the study; RS and YY performed the experiments; KF and YM organized the database; Sakurai M and YY performed the statistical analysis; Seishima M wrote the first draft of the manuscript. All authors contributed to manuscript revision, read and approved the submitted version.

\section{Conflicts of interest}

The authors declare that there are no conflicts of interest.

\section{Ethical approval}

The study protocol was approved by the ethics committee of Gifu University Graduate School of Medicine (2018-1949).

\section{Consent to participate}

The informed consent to participate in the study was obtained from all participants.

\section{Consent to publication}

Not applicable.

Availability of data and materials

Not applicable.

\section{Funding}

Not applicable.

\section{Copyright}

(C) The Author(s) 2021.

\section{References}

1. Szelest M, Walczak K, Plech T. A new insight into the potential role of tryptophan-derived AhR ligands in skin physiological and pathological processes. Int J Mol Sci. 2021;22:1104.

2. Kamata M, Tada Y. Safety of biologics in psoriasis. J Dermatol. 2018;45:279-86. 
3. Visser MJE, Venter C, Roberts TJ, Tarr G, Pretorius E. Psoriatic disease is associated with systemic inflammation, endothelial activation, and altered haemostatic function. Sci Rep. 2021;11:13043.

4. Dowlatshahi EA, van der Voort EA, Arends LR, Nijsten T. Markers of systemic inflammation in psoriasis: a systematic review and meta-analysis. Br J Dermatol. 2013;169:266-82.

5. Harden JL, Lewis SM, Lish SR, Suárez-Fariñas M, Gareau D, Lentini T, et al. The tryptophan metabolism enzyme L-kynureninase is a novel inflammatory factor in psoriasis and other inflammatory diseases. J Allergy Clin Immunol. 2016;137:1830-40.

6. Munn DH, Zhou M, Attwood JT, Bondarev I, Conway SJ, Marshall B, et al. Prevention of allogeneic fetal rejection by tryptophan catabolism. Science. 1998;281:1191-3.

7. Fallarino F, Grohmann U, You S, McGrath BC, Cavener DR, Vacca C, et al. The combined effects of tryptophan starvation and tryptophan catabolites down-regulate $\mathrm{T}$ cell receptor zeta-chain and induce a regulatory phenotype in naive T cells. J Immunol. 2006;176:6752-61.

8. Gostner JM, Becker K, Kofler H, Strasser B, Fuchs D. Tryptophan metabolism in allergic disorders. Int Arch Allergy Immunol. 2016;169:203-15.

9. Harden JL, Gu T, Kilinc MO, Rowswell-Turner RB, Virtuoso LP, Egilmezet NK, et al. Dichotomous effects of IFN-gamma on dendritic cell function determine the extent of IL-12-driven antitumor T cell immunity. J Immunol. 2011;187:126-32.

10. Gu T, Rowswell-Turner RB, Kilinc MO, Egilmez NK. Central role of IFNgamma-indoleamine 2,3-dioxygenase axis in regulation of interleukin-12-mediated antitumor immunity. Cancer Res. 2010;70:129-38.

11. Ito M, Ogawa K, Takeuchi K, Nakada A, Heishi M, Suto H, et al. Gene expression of enzymes for tryptophan degradation pathway is upregulated in the skin lesions of patients with atopic dermatitis or psoriasis. J Dermatol Sci. 2004;36:157-64.

12. Pantouris G, Serys M, Yuasa HJ, Ball HJ, Mowat CG. Human indoleamine 2,3-dioxygenase-2 has substrate specificity and inhibition characteristics distinct from those of indoleamine 2,3-dioxygenase-1. Amino Acids. 2014;46:2155-63.

13. Lowes MA, Suarez-Farinas M, Krueger JG. Immunology of psoriasis. Annu Rev Immunol. 2014;32:227-55.

14. Tian S, Krueger JG, Li K, Jabbari A, Brodmerkel C, Lowes MA, et al. Meta-analysis derived (MAD) transcriptome of psoriasis defines the "core" pathogenesis of disease. PLoS One. 2012;7:e44274.

15. Gudjonsson JE, Ding J, Johnston A, Tejasvi T, Guzman AM, Nair RP, et al. Assessment of the psoriatic transcriptome in a large sample: additional regulated genes and comparisons with in vitro models. J Invest Dermatol. 2010;130:1829-40.

16. Mitsui H, Suárez-Fariñas M, Belkin DA, Levenkova N, Fuentes-Duculan J, Coats I, et al. Combined use of laser capture microdissection and cDNA microarray analysis identifies locally expressed disease-related genes in focal regions of psoriasis vulgaris skin lesions. J Invest Dermatol. 2012;132:1615-26.

17. Suárez-Fariñas M, Fuentes-Duculan J, Lowes MA, Krueger JG. Resolved psoriasis lesions retain expression of a subset of disease-related genes. J Invest Dermatol. 2011;131:391-400.

18. Chiricozzi A, Guttman-Yassky E, Suárez-Fariñas M, Nograles KE, Tian S, Cardinale I, et al. Integrative responses to IL-17 and TNF- $\alpha$ in human keratinocytes account for key inflammatory pathogenic circuits in psoriasis. J Invest Dermatol. 2011;131:677-87.

19. Van der Leek AP, Yanishevsky Y, Kozyrskyj AL. The kynurenine pathway as a novel link between allergy and the gut microbiome. Front Immunol. 2017;8:1374.

20. Sakurai M, Yamamoto Y, Kanayama N, Hasegawa M, Mouri A, Takemura M, et al. Serum metabolic profiles of the tryptophan-kynurenine pathway in the high risk subjects of major depressive disorder. Sci Rep. 2020;10:1961.

21. Llamas-Velasco M, Bonay P, Concha-Garzón MJ, Corvo-Villén L, Vara A, Cibrián D, et al. Immune cells from patients with psoriasis are defective in inducing indoleamine 2,3-dioxygenase expression in response to inflammatory stimuli. Br J Dermatol. 2017;176:695-704. 
22. Nomura I, Gao B, Boguniewicz M, Darst MA, Travers JB, Leung DY. Distinct patterns of gene expression in the skin lesions of atopic dermatitis and psoriasis: a gene microarray analysis. J Allergy Clin Immunol. 2003;112:1195-202.

23. Mellor AL, Baban B, Chandler P, Marshall B, Jhaver K, Hansen A, et al. Cutting edge: induced indoleamine 2,3 dioxygenase expression in dendritic cell subsets suppresses $\mathrm{T}$ cell clonal expansion. J Immunol. 2003;171:1652-5.

24. DiNatale BC, Murray IA, Schroeder JC, Flaveny CA, Lahoti TS, Laurenzana EM, et al. Kynurenic acid is a potent endogenous aryl hydrocarbon receptor ligand that synergistically induces interleukin- 6 in the presence of inflammatory signaling. Toxicol Sci. 2010;115:89-97.

25. Mezrich JD, Fechner JH, Zhang X, Johnson BP, Burlingham WJ, Bradfield CA. An interaction between kynurenine and the aryl hydrocarbon receptor can generate regulatory $\mathrm{T}$ cells. J Immunol. 2010; 185:3190-8.

26. Julliard W, Fechner JH, Mezrich JD. The aryl hydrocarbon receptor meets immunology: friend or foe? A little of both. Front Immunol. 2014;5:458.

27. Fujii K, Yamamoto Y, Mizutani Y, Saito K, Seishima M. Indoleamine 2,3-dioxygenase 2 deficiency exacerbates imiquimod-induced psoriasis-like skin inflammation. Int J Mol Sci. 2020;21:5515.

28. Darlington LG, Forrest CM, Mackay GM, Smith RA, Smith AJ, Nicholas Stoy N, et al. On the biological importance of the 3-hydroxyanthranilic acid: anthranilic acid ratio. Int J Tryptophan Res. 2010;3:51-9.

29. Badawy AA. Hypothesis kynurenic and quinolinic acids: the main players of the kynurenine pathway and opponents in inflammatory disease. Med Hypotheses. 2018;118:129-38.

30. Takahashi H, Tsuji H, Hashimoto Y, Ishida-Yamamoto A, Iizuka H. Serum cytokines and growth factor levels in Japanese patients with psoriasis. Clin Exp Dermatol. 2010;35:645-9.

31. Solberg SM, Sandvik LF, Eidsheim M, Jonsson R, Bryceson YT, et al. Serum cytokine measurements and biological therapy of psoriasis-prospects for personalized treatment? Scand J Immunol. 2018;88:e12725.

32. Salimi ElizeiS, Poormasjedi-Meibod MS, Wang X, Kheirandish M, Ghahary A. Kynurenic acid downregulates IL-17/1L-23 axis in vitro. Mol Cell Biochem. 2017;431:55-65.

33. Cochez PM, Michiels C, Hendrickx E, Van Belle AB, Lemaire MM, Dauguet N, et al. AhR modulates the IL-22-producing cell proliferation/recruitment in imiquimod-induced psoriasis mouse model. Eur J Immunol. 2016;46:1449-59.

34. di Meglio P, Duarte JH, Ahlfors H, Owens NDL, Li Y, Villanova F, et al. Activation of the aryl hydrocarbon receptor dampens the severity of inflammatory skin conditions. Immunity. 2014;40:989-1001.

35. Opitz CA, Litzenburger UM, Sahm F, Ott M, Tritschler I, Trump S, et al. An endogenous tumour-promoting ligand of the human aryl hydrocarbon receptor. Nature. 2011;478:197-203.

36. Giustizieri ML, Mascia F, Frezzolini A, De Pità O, Chinni LM, Giannetti A, et al. Keratinocytes from patients with atopic dermatitis and psoriasis show a distinct chemokine production profile in response to T cell-derived cytokines. J Allergy Clin Immunol. 2001;107:871-7.

37. Kakinuma T, Saeki H, Tsunemi Y, Fujita H, Asano N, Mitsui H, et al. Increased serum cutaneous $\mathrm{T}$ cell-attracting chemokine (CCL27) levels in patients with atopic dermatitis and psoriasis vulgaris. J Allergy Clin Immunol. 2003;111:592-7.

38. Kwidzinski E, Bechmann I. IDO expression in the brain: a double-edged sword. J Mol Med (Berl). 2007;85:1351-9.

39. Vécsei L, Szalárdy L, Fülöp F, Toldi J. Kynurenines in the CNS: recent advances and new questions. Nat Rev Drug Discov. 2013;12:64-82. 